a conservative mechanical system interpreted by unitary operators in Hilbert space. Although these papers represent only a small part of his total contribution to mathematics, their quality is such that even if he had done nothing else, his place in mathematics would be assured.

A celebrated paper in 1933 solved Hilbert's fifth problem for the compact case and the solution was completed almost twenty years later by others to include the open $n$-dimensional groups. The question was (in modern terminology) whether in every locally Euclidean topological group there exist systems of co-ordinates such that the co-ordinates of the groupproduct $x y$ are analytic functions of the co-ordinates of $x$ and $y$. By an averaging process invented by him, von Neumann showed that the answer is 'yes' for a compact group.

Von Neumann's work on continuous groups include two papers in Volume 2 on almost periodic functions in a group.

Prof. Taub and those who have helped him are to be warmly thanked for editing these magnificent volumes. The publishers are to be complimented on the good printing and the modest price. Although none of von Neumann's papers is easy reading, the importance of his work is so great that these Collected Works must be given a place in all libraries concerned with modern mathematics.

Several mathematical colleagues have helped me with advice in the preparation of this review.

W. G. Penney

\section{BIOCHEMISTRY OF THE CARBOHYDRATES}

\section{Carbohydrates of Living Tissues}

By Prof. M. Stacey and Dr. S. A. Barker. Pp. xvii + 215. (Princeton, N.J.: D. Van Nostrand Company, Inc.; London: D. Van Nostrand Company, Ltd., 1962.) 45s.

$A \mathrm{~S}$ our knowledge of the chemistry of living A matter is extended, a study of the polysaccharide and oligosaccharide complexes present in the animal body is of paramount importance. The present book gives a comprehensive survey of these very complicated materials from the point of view of their known distribution, biological activity, constitution, and chemical properties, together with an account of any abnormalities which are known to occur as the result of a specific disease. The authors are to be congratulated for condensing into some two hundred pages most of the background and modern work on these substances. The bibliographies which conclude each of the chapters are such that the reader is at once presented with the major publications, up to and including 1960.

The first four chapters deal with glycogen, hyaluronic acid, chondroitin and chondroitin sulphate $A, B$ and $C$, and heparin and heparatin sulphate. Methods for the isolation, fractionation, detection and determination of each of these polysaccharides are detailed, and the chemical techniques utilized in the elucidation of their structures are outlined. The enzymatic pathways, in so far as they have been established, in the biosynthesis and degradation of these polymers are described, and the biological activity of each particular substance is stressed. An account is also given of changes in constitution and amount that occur as the result of a particular disease, and in the case of glycogen this is related to enzymatic deficiencies. The close structural relationship between hyaluronic acid and the chondroitin sulphates is excellently illustrated by the formula on p. 82 .

Keratosulphate, mucoitin sulphate, the neutral galactans, and a brief account of the lesser-known sulphated and phosphated polysaccharides, and the constitution, biological activity, and inhibition reactions of the human and cows' milk oligosaccharides form the subject-matter of Chapters 5 and 6 .

The last three chapters are devoted to the bloodgroup substances, to the mucoproteins of blood, urine, saliva, cerebrospinal and other fluids, and to the lipocarbohydrates respectively; in the latter are included the cerebrosides and gangliosides. The authors state that the book is presented only as an interim report, and this is particularly true of a number of substances described in the final chapters of which little is known other than the method of extraction, the constituent sugars and perhaps the biological activity.

The occasional footnote explaining a few of the medical terms could, with considerable advantage to the ordinary chemist, have been increased to include all such terminology, but this is a minor criticism. What is more important is that the book is well written, accurate, and attractively produced with clearly printed structural formulæ.

Inevitably much of the data is presented without comment, but the chief merit of the book is that it brings together a wealth of information which pre. viously has not been collected and which can otherwise only be obtained by an extensive search through the literature. At the published price this book is excellent value, and should immediately appeal to every scientist interested in the operation of the animal body, to biochemists, to research chemists, and to all engaged in the medical profession, particularly those studying chemical changes occurring in living tissues during illness.

Elizabetrh Peroival

\section{ORGANIC AND ENZYME- CATALYSED REACTIONS}

Mechanisms of Organic and Enzymic Reactions By S. G. Waley. Pp. xiii + 365. (Oxford : Clarendon Press ; London: Oxford University Press, 1962.) 70s. net.

R. S. G. WALEY has written a book in which the mechanisms of organic reactions and of enzyme-catalysed reactions are discussed together. $\mathrm{He}$ starts with molecular structure, and the general principles of mechanistic organic chemistry. He then considers in some detail the more or less usual list of reaction types which have yielded to mechanistic investigation, that is to say, nucleophilic substitutions, olefine-forming eliminations and their retrogressions, carbonyl reactions, saturated rearrangements and electrophilic aromatic substitutions. What distinguishes this from more conventional accounts of mechanism in organic chemistry is the inclusion of reactions, such as phosphate ester hydrolysis, which are of direct interest to biochemists, and of enzymatic reactions themselves. The author has therefore set himself the unenviable tasks of bringing enzymatic reaction mechanisms into the respectable fold of organic reaction mechanisms and, at the same 